\title{
Les fontainiers français des jardins de la Granja de San Ildefonso (1721-1772)
}

French fountain-makers in the gardens of the Granja de San Ildefonso

(1721-1772)

\section{Sophie Omère}

\section{(2) OpenEdition}

\section{Journals}

Édition électronique

URL : http://journals.openedition.org/artefact/476

DOI : $10.4000 /$ artefact.476

ISSN : 2606-9245

\section{Éditeur :}

Association Artefact. Techniques histoire et sciences humaines, Presses universitaires du Midi

\section{Édition imprimée}

Date de publication : 1 octobre 2016

Pagination : 259-272

ISBN : 978-2-7535-5174-9

ISSN : 2273-0753

\section{Référence électronique}

Sophie Omère, «Les fontainiers français des jardins de la Granja de San Ildefonso (1721-1772) », Artefact [En ligne], 4 | 2016, mis en ligne le 07 juillet 2017, consulté le 19 avril 2019. URL : http:// journals.openedition.org/artefact/476 ; DOI : 10.4000/artefact.476 


\section{Les fontainiers français des jardins de la Granja de San Ildefonso \\ (1721-1772)}

Sophie OMÈRE*

\section{Résumé}

La diffusion des techniques et du savoir-faire est un thème fort en matière d'hydraulique. Les recherches menées sur la communauté des fontainiers français au service du roi d'Espagne Philippe V permettent d'approfondir cette problématique. Installés à la Granja de San Ildefonso, dans la Sierra de Guadarrama, les fontainiers mettent en œuvre les innovations techniques déjà appliquées à Versailles et participent activement de la mise en scène du pouvoir royal. Cette étude de cas ouvre la réflexion sur les transferts techniques vers l'Espagne.

Mots-clés : fontainier, fonte, hydraulique, transferts.

\section{Abstract. French fountain-makers in the gardens of the Granja de San Ildefonso (1721-1772)}

The study about the community of French fountain-makers in Spain in the service of King Philip $V$ is to question the history of technology focusing on royal palaces hydraulics and the circulation of knowledge. The arrival of the French fountain-makers in la Granja de San Ildefonso changed the hydraulic technologies in Spain. From this perspective, hydraulics is an expression of political domination. This case study allows to investigate technological innovations and their transfers between France and Spain.

Keywords : cast iron, fountain-makers, hydraulics, transfers.

\footnotetext{
*. Historienne de l'art, Sophie Omère est actuellement conservatrice des monuments historiques à la DRAC Auvergne Rhône-Alpes. Elle a collaboré à trois ouvrages parus dans la collection " Patrimoines pour demain » (Éditions La passe du vent) en 2013 et 2015. Doctorante en histoire des techniques à l'EHESS depuis décembre 2013, elle s'intéresse au métier de fontainier et aux transferts techniques entre la France et l'Espagne au xvIII ${ }^{\mathrm{e}}$ siècle. Contact [sophie.omere@wanadoo.fr].
} 
Le 16 novembre 1700, le duc d'Anjou, petit-fils de Louis XIV, est proclamé roi d'Espagne sous le nom de Philippe V. La cérémonie d'intronisation au château de Versailles marque un tournant dans l'histoire politique espagnole avec l'instauration de la dynastie des Bourbons après presque deux siècles de règne de la maison de Habsbourg. Cette accession au pouvoir, rendue possible par le testament de Charles II d'Espagne qui désigne Philippe d'Anjou comme son héritier universel, est alors vivement contestée par les autres puissances européennes qui jugent que le trône devrait naturellement revenir au fils de l'empe-

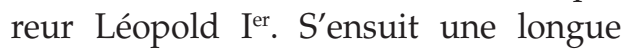
guerre qui ne se conclut qu'en 1713 par la ratification du traité d'Utrecht.

L'histoire de l'installation au pouvoir de Philippe V est parfaitement connue. Elle a été analysée sous plusieurs angles. Les travaux qui intéressent particulièrement notre étude portent sur les réformes menées par le roi et les moyens humains sur lesquels il s'appuie pour les mettre en œuvre. Durant les premières années du règne, le jeune roi s'entoure de conseillers français qui l'accompagnent dans son voyage de Versailles à Madrid. Dans sa thèse de doctorat, Catherine Désos dresse le portrait de ces Français installés dans les hautes sphères du pouvoir au début du règne $e^{1}$ et analyse leur rôle, majeur dans les changements profonds de la politique espagnole ${ }^{2}$. Plusieurs secteurs sont ainsi réformés : l'administration, les finances ou encore les armées. Le champ culturel ne fait pas exception. Les historiens se sont donc penchés sur la question des mutations et évolutions touchant au domaine artistique et culturel. Yves Bottineau fournit une somme exceptionnelle, qui fait encore autorité, sur l'art de cour de Philippe V, soulignant les divers courants et influences que les arts subissent tout au long du règne $e^{3}$. La question des transferts culturels est donc une problématique déjà bien connue et étudiée par les chercheurs, celle des transferts techniques l'est cependant beaucoup moins.

L'analyse du contexte de création des jardins du palais de la Granja de San Ildefonso et l'étude de sa fontainerie permettent d'élargir la question des transferts à l'étude de l'histoire des techniques. Le champ de recherche des transferts technologiques dans l'hydraulique a principalement été exploré entre l'Italie et la France, mais aussi entre l'Europe du Nord et la France. Pendant la Renaissance, les Italiens sont les grands spécialistes de la fontainerie. Ils diffusent leur art et technique à travers $l^{\prime}$ Europe. Sous le règne d'Henri IV, des hydrauliciens florentins attirés à la cour de France $s^{\prime} y$ installent pour diriger les travaux afférant aux fontaines ${ }^{4}$. La période suivante est marquée par des échanges entre l'Europe du Nord et la France dans le domaine des ouvrages hydrauliques de type canaux, digues, barrages et ponts ${ }^{5}$. Ces transferts s'instaurent en France dans le cadre d'une législation spéciale pour l'assèchement des marais ${ }^{6}$, concomitante à la réalisation d'opérations de dessiccation dont la direction est confiée à des ingénieurs hollandais?.

Tandis que $l^{\prime}$ hydraulique versaillaise au XVII ${ }^{e}$ siècle s'appuie sur une tradition de fontainerie déjà bien ancrée en France depuis le Moyen Âge, elle est, elle aussi, le fruit de plusieurs transferts technologiques d'horizons divers : d'un côté, 
l'Italie, avec en particulier l'influence florentine pour son goût de la mise en scène de la composition du jardin avec ses fontaines, et, de l'autre, le monde minier wallon et germanique avec sa machinerie. Qu'en est-il de l'hydraulique espagnole à l'arrivée de Philippe $\mathrm{V}$ au tout début du $\mathrm{XvIII}^{\mathrm{e}}$ siècle?

Dans l'historiographie espagnole, les études menées par Nicolás García Tapia montrent qu'il existe déjà, aux $x^{\prime} I^{e}$ et $\mathrm{XVII}^{\mathrm{e}}$ siècles une tradition d'utilisation de la mécanique hydraulique en Espagne. L'artificio de Juanelo, une machine construite pour élever les eaux du Tage à Tolède vers 1530 , ou la machine élévatoire des eaux du Pisuerga, conçue par Zubiaurre pour alimenter les jardins du palais du duc de Lerma à Valladolid au début du xvir siècle, sont autant de témoignages de l'existence d'inventeurs sur le territoire ibérique. Dans son analyse du manuscrit Los veintiún libros de los ingenios y de las máquinas, García Tapia, outre qu'il propose de l'attribuer à Pedro Juan de Lastanosa, aborde le thème de sa réception. Avant d'intégrer, en 1771, la Biblioteca Real, le manuscrit a appartenu à des ingénieurs et architectes, notamment à Juan Gómez de Mora et à Teodoro Ardemans qui considérait l'auteur comme l'égal des théoriciens Vitruve ou Alberti. Connu et probablement utilisé par les architectes, l'ouvrage traitait de sujets variés tels que les réservoirs, bassins, canaux, barrages, digues, moulins et machines hydrauliques incluant les ponts et les ports. García Tapia ajoute, sans plus de précision, que les architectes et fontainiers royaux mettent en application ces préceptes dans leurs travaux ${ }^{8}$. D'autres études plus récentes portent sur le système d'approvisionnement en eau de la ville de Séville et, notamment, l'analyse de l'évolution des caños de Carmona ${ }^{9}$ au xvirI ${ }^{\mathrm{e}}$ siècle. À l'arrivée de Philippe V, l'Espagne, s'appuyant sur une base solide de connaissances, est donc prête à s'approprier les savoir-faire techniques déjà maîtrisés pour l'essentiel et à les intégrer sur le chantier de la Granja de San Ildefonso.

Dans le domaine de l'art de la fontainerie, rappelons qu'à l'époque, les jardins du château de Versailles imaginés par le jardinier André Le Nôtre sont érigés en modèle et ce, très tôt après leur création. L'exploit, accompli par les ingénieurs et mathématiciens français, de conduire les eaux de diverses sources (détournement des eaux de la Bièvre, pompage des eaux de la Seine, captage et création d'étangs, etc.) au domaine royal de Versailles, émerveille toute $l^{\prime}$ Europe $^{10}$. Les maitres fontainiers n'ont probablement jamais bénéficié de la renommée des jardiniers, ingénieurs hydrauliciens ou architectes œuvrant à leurs côtés dans les jardins. Cependant, leur rôle ne se limitait pas au simple entretien des infrastructures d'adduction. Ils prenaient sans doute également part à la conception de la fontainerie. Il convient donc d'apporter des précisions sur ce métier peu connu et son rôle dans l'histoire des techniques au XVIII ${ }^{\mathrm{e}}$ siècle ${ }^{11}$.

Les descriptions littéraires des jardins de Versailles et leur diffusion renforcent la renommée de cette compétence spécifiquement française qui devient dès lors très recherchée. Dans le cas qui nous occupe, les sources d'inspiration de la Granja de San Ildefonso sont à rechercher du côté du domaine royal de Marly, à la fois dans son concept de création, un lieu de retraite pour le roi loin de l'étiquette 
de la cour, et dans sa réalisation, la plupart des sculpteurs envoyés en Espagne ayant travaillé sur le chantier de Marly ${ }^{12}$.

La place de choix accordée aux jeux d'eau et à leur diversité (miroirs d'eau, cascades, jets en lance, bouillons, etc.) participe de cette réputation et permet de mieux comprendre le contexte dans lequel le roi Philippe $\mathrm{V}$ choisit d'avoir recours à des fontainiers d'origine française pour mener à bien son grand œuvre à la Granja de San Ildefonso.

Pour examiner la question des transferts techniques vers l'Espagne, il nous semble important de présenter, pour commencer, le contexte de l'arrivée des artistes français à la cour de Philippe $\mathrm{V}$ et d'insister sur la mise en place d'une organisation hiérarchisée avec la création d'un service dédié aux fontaines. Notre analyse se concentre ensuite sur les différents savoir-faire techniques utilisés dans les jardins de la Granja et leurs origines. La partie suivante est consacrée à l'utilisation de ces techniques comme représentation du pouvoir. Il est à noter que cet article n'a pas vocation à répondre précisément à toutes les questions développées par ce sujet. Il pose des jalons et précise les pistes de réflexion à explorer, toujours en cours de recherche.

\section{La création de la fontainerie royale de la Granja de San Ildefonso}

Le palais et les jardins de la Granja de San Ildefonso, près de Ségovie, dans la Sierra de Guadarrama, sont construits selon le souhait du roi Philippe V. Il découvre les monts de Valsaín, situés sur le versant nord de la Sierra, lors de parties de chasse et choisit d'y implanter son palais, tant il est émerveillé par le paysage (illustration 5 , cahier couleur). L'occupation du site trouve ses origines dès le milieu $\mathrm{du} \mathrm{xv}^{\mathrm{e}}$ siècle lorsqu'Henri IV d'Espagne décide d'y établir un ermitage dédié à saint Ildephonse, archevêque de Tolède de la première moitié du vir ${ }^{\text {e }}$ siècle, auquel il voue un culte fervent. Le site est ensuite occupé par une congrégation de moines hiéronymites du monastère du Parral installé à Ségovie. Aucune installation hydraulique ne semble exister à cette époque.
La construction du palais et des jardins de la Granja reflète les origines du roi et son éducation reçue à la cour versaillaise. Le projet du palais est d'abord confié à l'architecte espagnol Teodoro Ardemans, tandis que la réalisation des jardins est placée sous la direction d'une équipe française. Dans son ouvrage de référence, Yves Bottineau analyse les raisons pour lesquelles architectes, peintres et sculpteurs français sont envoyés en Espagne à la demande du souverain. Le manque de grands maîtres sur place et les coutumes esthétiques appréciées par le roi et son entourage encouragent l'arrivée d'artistes français à la cour d'Espagne dès l'installation de la nouvelle cour. Dans le domaine le mieux connu, celui de la sculpture, Jeanne Digard mène une intéressante étude sur 
la collection de sculptures des jardins de la Granja de San Ildefonso ${ }^{13}$ en 1933. Elle analyse l'iconographie et la symbolique du programme sculpté de ces jardins tout en évoquant le travail et le parcours de leurs auteurs, choisis par l'Académie royale de peinture et de sculpture, institution française qui avait été chargée par Philippe V de désigner des artistes pour sa cour. Des autorisations de s'établir à l'étranger sont alors signées par le duc d'Antin qui dirige l'administration des Bâtiments du roi. Les artistes, pour certains, se rendent en Espagne dans un cadre bien défini pour une durée limitée; d'autres décident de s'y installer définitivement. Les chantiers d'envergure menés par le roi comme celui des jardins de la Granja de San Ildefonso sont des exemples éclatants de travaux dirigés par des Français. L'architecte René Carlier, élève du premier architecte de Louis XIV, Robert de Cotte, et le jardinier Étienne Boutelou sont en charge du dessin des jardins. Leurs activités et réalisations avant leur installation en Espagne sont peu documentées. Le terrassement et la direction des travaux sont conduits par l'ingénieur militaire Étienne Marchand. Une équipe de sculpteurs est spécialement missionnée pour la réalisation des groupes sculptés et des fontaines. La création du réseau hydraulique est, elle aussi, mise en œuvre par des fontainiers français. Le réseau se constitue progressivement au gré des achats et acquisitions de nouveaux terrains par le roi. Initié en 1721, il se poursuit jusqu'en 1743 avec la construction des jeux d'eau de la fontaine des Baños de Diana (illustration 6, cahier couleur) dont l'achèvement date de 1745 .
Le dépouillement des fonds de l'Archivo general de Palacio permet de reconstituer l'histoire de la mise en place de la fontainerie royale de la Granja de San Ildefonso et de l'arrivée des fontainiers français qui se succèdent à sa tête $^{14}$. L'Archivo general de Palacio a été créé en 1814 avec pour objectif de recueillir, classer et conserver tous les documents produits par les différents départements et institutions chargés du gouvernement ou de l'administration de la Maison royale et du patrimoine de la Couronne ${ }^{15}$. Les fonds documentaires dépouillés appartiennent aux sections Administraciones patrimoniales et Personal.

Le principe de fonctionnement du système hydraulique des jardins de la Granja repose sur la présence d'un réservoir, el Mar, situé sur le point culminant du jardin à près de 1250 mètres d'altitude et d'une capacité de plus de $215000 \mathrm{~m}^{3}$. Huit autres réservoirs, disséminés aux quatre coins du jardin et installés à des hauteurs différentes, complètent l'alimentation en eau des fontaines. Leur emplacement permet d'obtenir les pressions adéquates pour bénéficier de la hauteur désirée pour chaque jet d'eau. Les jardins comptent au total une vingtaine de fontaines et un canal, la Ría, dans lequel se déversent les eaux d'une partie des fontaines. Chaque fontaine est dotée d'une chambre de vannes destinées à recevoir les clefs d'ouverture des jeux d'eau. S'ajoute à cela, pour la plupart des fontaines, l'existence de clés destinées à réguler la portée des jets d'eau obliques. Tant pour les réservoirs que pour les fontaines, le système de vidange est actionné par une décharge de fond, dont l'ouverture est bouchée d'une soupape (illustration 7, cahier couleur). La circulation 
des eaux dans les bassins est protégée des impuretés et corps étrangers par une armature de feuilles de plomb perforées servant de filtre (illustration $\mathbf{8}$, cahier couleur). Les conduites principales sont dotées d'un système de ventilation.

L'ensemble de ce système hydraulique a été conçu et entretenu par la fontainerie royale. La première mention de la présence d'un fontainier sur le chantier de la Granja de San Ildefonso apparaît dans la comptabilité des «travaux des Jardins Royaux » de mars $1721^{16}$. À la date du 15 mars, arrive sur le chantier un certain François Dorléans ${ }^{17}$, présenté comme «fontainier qui se doit de conduire la formation de la tuyauterie et des fontaines de ces dits travaux ». Aucun document dans les archives consultées ne nous a permis de définir ou d'émettre quelque hypothèse plus précise concernant ses origines.

Le chantier de fontainerie se met alors progressivement en place sous le contrôle d'un premier fontainier (fontanero mayor) et de son second (ayudante de fontanero), tous deux français. François Dorléans et Antoine Chapotto ${ }^{18}$ forment le premier tandem.

La comptabilité fait par ailleurs apparaître la présence de plusieurs Français dans l'équipe de fontainerie à laquelle viennent se joindre des Espagnols. Leur nombre varie sur la période. Le dépouillement effectué a permis de connaître la composition de la fontainerie sur une cinquantaine d'années et de dresser un tableau des employés sur la période étudiée. Il est complexe de déterminer avec précision les nationalités des employés de la fontainerie, certains noms étant traduits en espagnol. Jusqu'en 1760, la direction de la fontainerie est assurée par deux Français, le premier fontainier et son aide. Parmi les fontainiers " ordinaires », seulement deux sont identifiés comme Français. Le dernier d'entre eux s'éteint en 1735.

À la mort des premiers fontainiers " en chef ", d'autres fontainiers de nationalité française sont appelés, tour à tour, à diriger le chantier. Cette situation perdure jusqu'au décès de François Guillaume Desjardins, qui intervient le 27 juin $1772^{19}$, après trente-quatre ans à la tête de l'office.

L'analyse de la suppression du poste de premier fontainier à San Ildefonso donne quelques clefs pour comprendre les raisons prévalant au recrutement des Français.

À la mort de Desjardins, nous savons que c'est un Espagnol, le premier oficial Lorenzo Sanchez, qui obtient le titre de premier fontainier de San Ildefonso. Une note rédigée après sa mort en 1790 explique les conditions de sa nomination : « à la mort de François Desjardins en 1772, Sa Majesté avait ordonné de supprimer la place de premier fontainier puisque l'on ne pensait pas augmenter les nouveaux jeux d'eau dans les fontaines du jardin et que pour conserver en bon état celles existantes il suffisait qu'en soit chargé Lorenzo Sanchez ${ }^{20}$. » Cette note permet déjà de retenir la différence essentielle entre le processus de création (formulé sous le terme $d^{\prime}$ " augmentation » ici) des effets d'eau et les interventions d'entretien. Ce sont deux opérations nécessaires dans le jardin mais qui ne supposent pas les mêmes connaissances en matière de fontainerie. "Ces oficiales n'ont pas la capacité d'inventer de nouvelles fontaines, je les trouve capable de par leur grande expérience pratique de 
maintenir ce qui existe en bon état ${ }^{21}$ ", comme le rappelle l'intendant Cavallero. La question de l'invention de la fontaine, qui revient à plusieurs reprises dans les archives, est un élément essentiel pour comprendre le rôle des fontainiers français en Espagne. Rappelons que la commande de la dernière fontaine créée à la Granja est passée en octobre 1737. Le gros œuvre et le système de jeux d'eau sont achevés dès 1743, mais ce n'est qu'en 1745 qu'elle est pleinement terminée et mise en fonctionnement.

Après avoir rapidement évoqué la composition de la fontainerie dirigée par les Français, il convient maintenant d'aborder le sujet du point de vue des techniques et des savoir-faire.

\section{À la recherche d'une expertise : les fontainiers français en Espagne}

L'installation des fontainiers français à la Granja de San Ildefonso a permis la mise en œuvre en Espagne de procédés techniques de fontainerie utilisés sur les chantiers royaux français. Il semblerait qu'avant leur arrivée, la fontainerie s'inspire encore en grande partie des réalisations techniques de la Renaissance $^{22}$. Un bilan sur les savoirfaire hydrauliques dans les jardins espagnols avant l'arrivée de Philippe $\mathrm{V}$ au pouvoir est en cours d'étude. Le Jardín de la Isla à Aranjuez, dont le programme de création des fontaines réalisé sous la direction de l'architecte Juan de Herrera date de 1582, en est un bon exemple. Ce jardin est représentatif de la typologie de jardins « à l'italienne » qui se développe dans la péninsule ibérique sous l'impulsion de Philippe II. Le tracé du jardin s'organise autour d'un axe central entouré de compartiments rectangulaires, eux-mêmes divisés en carrés. Les intersections des axes centraux sont marquées par des placettes dotées de fontaines. Leur disposition en ligne droite permet de faciliter leur alimentation en eau. De petits jets d'eau disposés au sol venaient surprendre les visiteurs dans la pure tradition italienne visant à ménager des effets et surprises hydrauliques en cours de parcours. Ce jardin alliait différentes influences, l'intimité des jardins islamiques avec des fontaines basses, l'ordonnancement géométrique, les jeux d'eaux et l'allusion mythologique des jardins maniéristes italiens et parterres bas de fleurs à la manière flamande très appréciés par Philippe II $^{23}$.

La fontainerie prend également ses sources dans des traditions plus anciennes dont les témoignages sont encore très présents sur le territoire (vestiges de l'époque romaine et de la période musulmane) et sur des expérimentations d'hydrauliciens espagnols durant les $\mathrm{XVI}^{\mathrm{e}}$ et XVII ${ }^{\mathrm{e}}$ siècles.

L'organisation du travail, la répartition et les compétences des ouvriers œuvrant dans les jardins semblent confirmer le fait que les installations hydrauliques étaient modestes. Avant l'arrivée des Français, les employés qui s'occupent du jardin traitent également des problé- 
matiques liées à la fontainerie. Dans les comptes d'Aranjuez, jardiniers et fontainiers ne sont pas différenciés ${ }^{24}$. Plus tard, apparaît l'intitulé de jardinero fontanero : un document présente l'équipe des jardins d'Aranjuez exposant le parcours de chacun ${ }^{25}$. La plupart des jardiniers fontainiers ont débuté leur carrière comme ouvriers attachés au jardin. Parmi les soixante-quinze jardiniers, fontainiers et botanistes ordinaires comptabilisés dans les jardins d'Aranjuez le 21 mai 1742, figurent six officiers relevant de la catégorie des jardiniers-fontainiers. Avant d'obtenir la place de jardinero fontanero, ils sont quatre à avoir occupé le poste d'ouvrier de jardin pendant respectivement cinq, sept, quatre et sept ans.

Le « renouveau » dans les techniques hydrauliques semble ainsi coïncider avec l'arrivée des fontainiers français sur le chantier de la Granja de San Ildefonso et la mise en place d'un réel office de fontainerie doté de son propre système hiérarchisé. La première organisation réglementée de la fontainerie qui nous est parvenue est connue par le document de $1733^{26}$, rédigé par le premier fontainier Maimpon de la Roche. Gouvernée par le premier fontainier et son aide, la fontainerie n'est alors composée que de cinq fontainiers. Quelques années plus tard, un document conservé dans les ordres royaux, daté de septembre $1738^{27}$, donne l'image de la fontainerie en pleine expansion. Elle se compose alors d'une Plana de la Familia ou Plana mayor ${ }^{28}$, à laquelle appartiennent le premier fontainier, son premier aide et deux oficiales. Vient ensuite la Plana menor de fontaneros y peones arreglados, qui comprend deux fontainiers oficiales, quatre fontainiers, un secrétaire, un charpentier et cinq ouvriers qualifiés (peones arreglados). La composition de la fontainerie a donc complètement évolué en cinq ans : de sept employés en 1733 , elle passe à dixsept personnes fin 1738 .

Pourquoi faire appel à l'expertise des Français? Quelles sont donc les connaissances techniques transmises par ces derniers? En préambule, il convient de rappeler que les problématiques qu'ils rencontrent à la Granja sont bien différentes de celles des chantiers royaux français. L'implantation du palais de la Granja au pied d'une montagne en fait un terrain propice à la création de jeux d'eau et de fontaines. Le tracé en forte pente des jardins a été conditionné par la topographie naturelle du site. La présence de sources et d'eau en abondance est un atout majeur : les ruisseaux Morete, Carneros et Chorranca permettent l'alimentation de l'ensemble du système hydraulique. Contrairement à Versailles où l'essentiel du travail des fontainiers et des ingénieurs s'était porté sur la recherche de l'eau, son stockage dans des réservoirs et son acheminement jusqu'aux jardins, celui de la Granja va consister à tirer profit de la déclivité du terrain et de ses ressources naturelles.

La technique hydraulique utilisée sur l'ensemble des jardins est dite gravitaire $^{29}$. Les réflexions menées par les architectes et les fontainiers reposent alors sur des calculs visant à déterminer les emplacements, l'altitude et la capacité les plus appropriés des réservoirs et étangs artificiels qui alimentent les fontaines, le diamètre des tuyaux et la forme des ajutages, afin d'obtenir la hauteur de jets d'eau souhaitée. Ces expérimentations sont précieusement compilées dans les divers traités d'hydraulique des XVII ${ }^{\mathrm{e}}$ 
et $\mathrm{XVIII}^{\mathrm{e}}$ siècles qui établissent des tables de référence. Parmi les fontaines de la Granja, celle de Las Ranas présente un impressionnant répertoire de jeux d'eau. Pour ce faire, elle ne possède pas moins de soixante-quatre jets d'eau dont vingtquatre verticaux et quarante obliques permettant la tenue de trois effets d'eau différents.

L'utilisation de l'hydraulique gravitaire dans les jardins de la Granja ne peut être considérée comme une nouveauté. A contrario, l'introduction d'un nouveau type de canalisations dans le réseau hydraulique est capitale. Une des principales innovations du chantier versaillais résidait dans l'emploi d'un matériau inédit, la fonte. Comme l'explique Éric Soullard dans sa thèse, la mise en place de tuyaux de fonte à brides et à vis, dont les premiers tests de fabrication sont réalisés dans les années 1670, constitue une vraie révolution à Versailles. À la Granja comme à Versailles, la solidité de ces nouvelles conduites permet d'éviter les fuites et les réparations incessantes nécessitées par les traditionnels tuyaux de bois, terre cuite ou cuivre utilisés auparavant et plus sujets à la casse lorsqu'ils sont soumis à de fortes pressions (illustration 9, cahier couleur). L'emploi du plomb se perpétue dans certaines zones stratégiques du réseau. Sa malléabilité naturelle se prête parfaitement à la conception des bifurcations ou des jonctions avec les soupapes. Il est également présent à l'intérieur des fontaine ${ }^{30}$. Les tuyaux de plomb étaient fabriqués par les fontainiers à partir de feuilles de plomb enroulées sur un cylindre et soudées entre elles par un petit filet de plomb fondu coulé le long de la jointure du tuyau afin d'obtenir son étanchéité. Cette spécialisation est à associer à la formation des fontainiers liés à la communauté des maîtres plombiers-fontainiers. Mais les tuyaux de fonte constituent l'essentiel du réseau : sur les cinquante-trois conduites de fonte et de plomb du XVIII ${ }^{\mathrm{e}}$ siècle, de longueur et de section variées, aujourd'hui encore conservées dans les jardins, près de $87 \%$ sont en fonte ${ }^{31}$. La récente restauration du système hydraulique a permis de préserver, consolider et conserver le maximum des matériaux d'origine.

L'emploi des canalisations en fonte, généralisé sur la quasi-totalité du réseau hydraulique, est donc la nouveauté technique transmise par les Français qui comprend deux volets : celui de l'approvisionnement en matière première et celui de la transformation et fabrication des tuyaux. La question de l'approvisionnement en tuyaux de fonte des chantiers des jardins de la Granja est en cours d'étude. Certaines découvertes sur le sujet permettent dès à présent d'avancer quelques hypothèses. L'approvisionnement s'effectuait pour partie depuis la France. Des raisons techniques, à savoir les innovations expérimentées sur le chantier versaillais, expliqueraient ce choix. Les dépouillements dans les archives apportent des précisions. Des commandes sont passées pour l'importation de " 384 tuyaux de fonte, 164 de 4 pieds de large et 14 pouces de diamètre, 9 de 4 pieds et 10 pouces de diamètre "de la manufacture de France ${ }^{\prime \prime 32} »$. D'autres traitent de matériaux en provenance de "manufactures de Hollande ou de France ${ }^{33} »$.

Nous savons par ailleurs qu'au moins une partie $\mathrm{du}$ fer provient directement d'Espagne, des sites d'exploitation de 
Liérganes et de la Cavada, situées en Cantabrie, au nord du pays. Des ateliers de fonte, premiers de la péninsule ibérique à être dotés de hauts fourneaux, $s^{\prime} y$ installent durant le premier tiers du $\mathrm{XVII}^{\mathrm{e}}$ siècle. Ils sont alors exclusivement destinés à la fabrication de pièces d'artillerie. Entre 1716 et 1800, ces deux manufactures connaissent une période de forte activité, due notamment à l'expansion du nombre des traversées de l'Atlantique et aux années fastes de l'Armada espagnole qui protège les bateaux en partance pour la route des Indes. Sous la direction de Nicolás Xavier de Olivares, la production atteint des sommets. C'est à cette même époque qu'y sont conçus les tuyaux des fontaines d'Aranjuez et de San Ildefonso, dont le volume de fonte occupe une part très importante de leur production. La mention de la présence d'un entrepreneur français sur le site de Liérganes « pour superviser à la fonte des conduits pour les fontaines ${ }^{34}$ » reste encore à élucider. Quel est son rôle? Comment supervise-t-il les travaux? Les Français, fontainiers et entrepreneurs, semblent avoir un rôle de passeurs, de vecteurs de transmission d'un savoir-faire technique dont la maîtrise existe en Espagne mais est davantage exploitée dans le reste de l'Europe.

Les découvertes réalisées au cours de dépouillements d'archives et les hypothèses qui en découlent tendent à prouver l'importance de la présence des fontainiers français en Espagne. Comme à Versailles, les sciences et techniques sont au service du pouvoir royal.

\section{La fontainerie au service du pouvoir}

Philippe d'Anjou naît à Versailles en 1683, il est donc totalement empreint de cette culture centrée autour de la figure $\mathrm{du}$ monarque. Les arts et techniques sont utilisés à son profit. Les innovations contribuent à magnifier sa puissance et son aura. En cela, il s'inscrit également dans la droite lignée de ses prédécesseurs de la Maison de Habsbourg.

Dès son accession au trône, le roi montre son peu de goût pour l'exercice du pouvoir. Son état de santé et son caractère dépressif le conduisent à rechercher un lieu au sein duquel il pourra se réfugier à l'abri des contraintes du pouvoir. Son choix se porte sur le site de la Granja de San Ildefonso. Il pense un temps réussir à s'y installer pour mener une vie d'ascète et abdique au profit de son fils le 15 janvier 1724 . Son souhait ne sera exaucé que quelques mois : le nou-

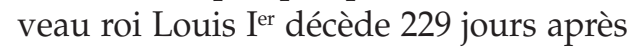
le début de son règne et Philippe $\mathrm{V}$ est alors obligé de reprendre les rênes du pays.

À la Granja, sa volonté d'aménager des jardins aux jeux d'eau somptueux traduit ses racines françaises. Il cherche à rivaliser, voire à surpasser son aïeul, et à manifester son pouvoir. Pour arriver à ses fins, le roi est obligé de faire appel aux compétences nécessaires à la réalisation de son projet. Les fontainiers français ayant travaillé sur les chantiers du roi (Versailles, Marly) ou de son entourage (Chantilly) sont réputés posséder les 
secrets techniques permettant d'obtenir de somptueux effets d'eau. Philippe V cherche donc à attirer ces techniciens dans son royaume. Les différents effets d'eau des fontaines de la Granja impressionnent par leur hauteur, la variété de dessins et la durée du spectacle offert aux yeux des visiteurs avant de disparaître. Les événements créés autour de ces fontaines sont autant des démonstrations de l'immense pouvoir du roi que de son contrôle sur les éléments naturels. Les thèmes mythologiques auxquels elles font référence sont empreints de significations symboliques. Le roi est considéré comme le sauveur de la monarchie, victorieux de ses ennemis de la Guerre de Succession. C'est le cas pour la fontaine d'Andromède de la Carrera de los cabellos. Dans ce groupe sculpté, Andromède représente la monarchie espagnole sauvée par Persée (le roi Philippe V), aidée de Minerve symbolisant la monarchie française.

L'étude des ouvrages anciens des XVIII et $\mathrm{XIX}^{\mathrm{e}}$ siècles tend à prouver que le défi relevé par le roi est une belle réussite ${ }^{35}$. Plusieurs visiteurs contemporains de la création des jardins en témoignent. SaintSimon, alors ambassadeur en Espagne, visite le site de la Granja de San Ildefonso en 1722. Les jardins qu'il décrit sont encore en grande partie en chantier, mais se dessinent déjà les grandes lignes d'un projet prometteur. Dans ses Mémoires ${ }^{36}$, l'ambassadeur critique vivement le site dans lequel le roi a choisi d'installer son palais, mais il semble apprécier la variété, la force et le nombre imposant de bassins et d'effets d'eau des premiers ouvrages déjà en place et de ceux à venir. Cette description ne nous permet pas de juger de la magnificence des jeux d'eau.
D'autres récits plus tardifs de voyageurs étrangers (anglais, français ou italiens) et espagnols en offrent le témoignage ${ }^{37}$. Citons, par exemple, le Nouveau voyage en Espagne fait en 1777 et 1778 de JeanFrançois Peyron et celui de Jean-François Bourgoing, publié en 1788 à Paris en trois volumes. Peyron expose son avis sur les jardins, décrit les éléments qu'il juge médiocre et fait l'apologie des autres. À l'instar des autres voyageurs, il compare La Granja et Versailles :

« Il [Philippe V] vouloit avoir le portrait de Versailles en miniature; et pour qu'il ressemblât davantage, il choisit une assiette stérile, mais superbe par les effets naturels dont il était susceptible. Les eaux surtout, aussi claires et limpides que celles de Versailles sont troubles, ne contribuent pas peu à rendre ce jardin une des plus belles situations de la terre. [...] Le bain de Diane est un chef-d'œuvre d'hydraulique; les eaux s'échappent par cent bouches, et retombent avec un bruit terrible; la vapeur qui s'en exhale répand à cinquante pas à la ronde une douce fraîcheur dans les allées. »

C'est par le biais de la peinture également que nous pouvons admirer ces ouvrages hydrauliques. En octobre 1821, le peintre Fernando Brambilla reçoit la commande royale de représenter des vues de San Ildefonso. Comme le précise José Luis Sancho, ces vues sont dominées par les jeux d'eau. Ils sont les vrais « protagonistes [...] conférant toujours une valeur très secondaire à la sculpture ${ }^{38}$ ". C'est bien la prouesse technique opérée par les hydrauliciens français qui est à 
l'origine de cette grande réussite esthétique et artistique, parfaitement bien illustrée dans cette série de peintures des jardins de la Granja.

L'étude du phénomène de migration de certains fontainiers français en Espagne sous le règne de Philippe $\mathrm{V}$ permet d'interroger l'histoire des techniques en matière d'hydraulique princière et la circulation des savoirs entre France et Espagne à une période où des liens étroits se sont tissés entre la cour madrilène et celle de Versailles.

La problématique de diffusion des techniques et du savoir-faire est un thème fort dans le domaine de l'hydraulique. Cette étude permet d'ouvrir la réflexion aux transferts techniques vers l'Espagne pour lesquels il existe peu d'études. Citons pour exemple le cas de l'ingénieur espagnol aux origines françaises, Augustin Bétancourt, étudié par Irina Gouzévitch ${ }^{39}$. Cet expert, reconnu pour ses compétences techniques, est missionné aux quatre coins de l'Europe tout au long de sa carrière. Après avoir étudié à l'École des ponts et chaussées de Paris, il crée, en 1802, une école sur ce même modèle à Madrid ${ }^{40}$. Les études sur les transferts techniques dans l'aire méditerranéenne représentent un enjeu important puisqu'elles permettent d'aborder la problématique d'autres centres ou pôles d'innovation en Europe.

L'état de l'hydraulique espagnole à l'arrivée de Philippe $\mathrm{V}$ au tout début du XviII ${ }^{\mathrm{e}}$ siècle conjugue alors de nombreuses influences à l'image de l'hydraulique princière française du XvII ${ }^{\mathrm{e}}$ siècle.

Dans l'optique des grandes réformes d'ordre administratif, économique et artistique que connaît l'Espagne au moment de l'installation à sa tête de la
Maison de Bourbon, les chantiers d'hydraulique, expression de la domination politique, occupent une place importante. Leur étude permet d'approfondir les problématiques portant sur la symbolique de faste et de puissance associée aux jeux d'eau de formes variées et sur l'utilisation d'innovations technologiques et leurs transferts dans un contexte particulier impliquant l'installation d'un nouveau réseau de techniciens spécialisés, des fontainiers français. L'impact réel de leur présence en Espagne et leur rôle dans le renouvellement, sur le long terme, des techniques d'hydraulique dans le pays doivent encore être précisément définis.

\section{Notes}

1. Catherine Désos, Les Français de Philippe V. Un modèle nouveau pour gouverner l'Espagne (17001724), Strasbourg, Presses universitaires de Strasbourg (Sciences de l'histoire), 2009.

2. Ibid., p. 462 ; C. Désos ne fait qu'une très brève mention de trois fontainiers français, éléments repris de l'ouvrage d'Yves Bottineau.

3. Yves Bottineau, L'art de cour dans l'Espagne de Philippe V (1700-1746), Bordeaux, Féret, 1962; nouvelle édition corrigée et augmentée, Sceaux, Mémoires du musée de l'Ile-de-France, vol. $n^{\circ} 1$, 1993.

4. Création en 1599 d'une surintendance des fontaines des Bâtiments du roi.

5. Raphaël Morera, L'assèchement des marais en France au XVII siècle, Rennes, PUR, 2011.

6. Quatre édits royaux sont publiés entre 1599 et 1613.

7. L'ingénieur brabançon Humfroy Bradley, chargé de nombreux chantiers, est à l'origine de la création de l'Association pour l'assèchement des lacs et marais de France en 1605. L'assèchement des plaines d'Arles et de Tarascon est dû à l'intervention de l'ingénieur hollandais Van Ens en 1642.

8. Nicolás García TAPIA, "Ciencia y técnica en la España de los Austrias : una visión desde la perspectiva de las investigaciones », Cuadernos de historia moderna, $\mathrm{n}^{\circ} 15,1994$, p. 199-214. 
9. Manuel F. Fernandez Chaves, Los caños de Carmona y el abastecimiento de agua en la Sevilla moderna, Séville, EMASESA Metropolitana, 2011.

10. Ce thème est l'objet de la thèse de doctorat d'Éric Soullard, Les eaux de Versailles (XVII XVIII ${ }^{e}$ siècles), sous la direction de Gérard Sabatier, université Pierre Mendès France de Grenoble, 2011.

11. Sur les fontainiers à Versailles au $\mathrm{XVII}^{\mathrm{e}}$ siècle, Chiara SANTINI, « Les artistes de l'eau. Fontainiers à Versailles au Grand Siècle ", Projets de paysage [en ligne], mis en ligne le 23 décembre 2009 [http:/ / www.projetsdepaysage.fr/fr/les_artistes_de_1_ eau].

12. María Jesús Herrero SANZ, « Los jardines de la Granja de San Ildefonso : Felipe V entre Marly y Versalles ", Bulletin du Centre de recherche du château de Versailles [en ligne], 2012, mis en ligne le 18 décembre 2013 [http:/ / crcv.revues.org/11940].

13. Jeanne DigARD, Les jardins de La Granja et leurs sculptures décoratives, La Granja, Leroux, 1934.

14. Ces informations sont tirées du rapport de la bourse Carnot 2014 de Sophie OMÈre, La fontainerie des jardins de la Granja de San Ildefonso, décembre 2015, non publié, partie I du rapport.

15. Margarita GonzÁlez CRIstóbAl, « Archivo general de Palacio », Arbor, CLXIX (665), mai 2001, p. 267-286.

16. Archivo general de Palacio [ensuite AGP], Adm. patrimoniales, Real Sitio de San Ildefonso [ensuite RSSI], caja 23129 expediente [ensuite exp.] 1 : « Nomina de los jornales de los maestros y oficiales, peones y demás personas que trabajan y asisten en la obra de los Jardines, marzo 1721 »; cette liste indique le salaire perçu par chaque employé sur le chantier.

17. AGP, Adm. patrimoniales, RSSI, caja 23129 exp. 1 : «A M. Francisco Orleans se le debe pagar quinientos y diez reales de vellón desde el día quince de marzo hasta oydía día de la fecha inclusive a razón de diez reales en cada uno que le tengo señalados como â fontanero que ha de asistir para la formación de cañerías y fuentes en dicha obra ».

18. Le 23 juin apparaît pour la première fois le nom d'un autre fontainier, d'origine française, Antoine Chapotto (AGP, Adm. patrimoniales, RSSI, caja 23129 exp. 1).

19. AGP, Adm. patrimoniales, RSSI, caja 23401 : acte notarié.

20. AGP, Personal, caja 16647 exp. 12 : «se informó en el año de 1777 por el Intendente Don Agustín Caballero, que a la muerte de Don francisco Desjardins en el año de 1772 había mandado SM suprimir la plaza de fontanero mayor respecto de que no se pensaba aumentar nuevos juegos de aguas en las fuentes del jardin y que para conservar en buen estado las existentes bastaba se encargase de ello Sanchez, asignándosele por este trabajo una ayuda de costa de 1500 reales. En consecuencia de este informe se decretó en 18 de octubre de 1777 que SM concedía à Lorenzo Sanchez solo el titulo de fontanero mayor sin el empleo ni el sueldo en cuya virtud podría usar de aquel; y que por la Pagaduría se le diese por una vez una gratificación de 800 reales ».

21. AGP, Personal, caja 16843 exp. 27, lettre du 27 juin 1772.

22. Entretien avec Luis Sala Santa Ana, ingénieur hydraulicien retraité au Departamento de arquitectura y jardines du Patrimonio nacional.

23. José Luis SAncho Gaspar, Guía Real Sitio de Aranjuez, Madrid, Patrimonio nacional, 2014, p. 83.

24. Comptes antérieurs à 1738.

25. AGP, Adm. patrimoniales, Real Sitio de Aranjuez (RSA), caja 14154 : Relation de 1742 sur les 75 jardiniers, fontainiers et fleuristes ordinaires.

26. AGP, Adm. patrimoniales, RSSI, caja 23829 exp. 1.

27. AGP, Adm. patrimoniales, RSSI, caja 23830 exp. 2.

28. La Plana mayor comprend les principaux officiers au service de la famille royale, les hauts responsables.

29. Le fonctionnement des fontaines est basé sur la loi de la gravité.

30. Une étude historique, scientifique et technique a été menée sur le système hydraulique des jardins de la Granja de San Ildefonso; Carlos GuZMÁN AzcÁRATE, «Estudio sobre la recirculación del agua de las fuentes del palacio de la Granja de San Ildefonso (Segovia) ", Ingeniería civil, $\mathrm{n}^{\circ} 122$, 2001, p. 61-72.

31. Ibid.

32. AGP, Adm. patrimoniales, RSSI, caja 23832 exp. 1 : «384 canos de hierro, 164 de 4 pies de largo $y$ 14 pulgadas de diámetro, 9 de 4 pies y 10 pulgadas de diámetro "de la Fabrica de Francia" ".

33. AGP, Adm. patrimoniales, RSSI, caja 23040 exp. 2 : «Para la nueva dirección que se da en el sitio de Aranjuez a la cañería que pasaba debajo de la Ballestería, se necesitan doce cañones de hierro de tres pulgadas Francesas de diámetro, y tres pies y medio Franceses de largo de los de à cuatro tornillos y de las Fabricas de Holanda, o Francia».

34. AGP, Adm. patrimoniales, RSSI, caja 23155 exp. 1: comptabilité des années 17211723.

35. Y. Bottineau, L'art de cour dans l'Espagne de Philippe V..., op. cit., p. 431 : poème de l'abbé Delille. 36. T. 19, chapitre XI.

37. Parmi les voyageurs étrangers, l'italien Norberto Caimo et l'anglais Henry Swinburne décrivent les jardins de la Granja dans des ouvrages publiés dans le dernier quart du XVIII ${ }^{\mathrm{e}}$ siècle. 
38. José Luis SANCHO, Las vistas de los Reales Sitios por Brambilla : La Granja de San Ildefonso, Madrid, Patrimonio nacional, 2000, p. 30.

39. Irina GouzÉvitch, « Les voyages en France et en Angleterre et la naissance d'un expert technique : le cas d'Augustin Betancourt (1758-1824) ",
Documents pour l'histoire des techniques [en ligne], 19 | 2e sem. 2010, 10/06/2011 (consulté le 22 novembre 2015).

40. Actuelle Escuela técnica superior de ingenieros de caminos, canales y puertos de Madrid. 